\title{
De "la folie qui serait venue dans les flancs des bateaux négriers"
}

\author{
Da "loucura que teria vindo nos flancos dos navios negreiros"
}

\author{
Maria Fernanda Arentsen
}

Université de Saint-Boniface - Canadá $\diamond$

\begin{abstract}
Résumé: Ce travail propose une lecture du Livre d'Emma, publié en 2001, roman de Marie-Célie Agnant qui a fait l'objet de plusieurs études critiques suivant diverses approches, notamment postcoloniale et féministe. Cependant, la folie et l'institutionnalisation d'Emma méritent une attention particulière. Nous nous attarderons donc sur ces aspects du roman, en le situant dans les contextes de la réflexion foucaldienne sur le biopouvoir et de la folie dans le discours littéraire. Nous étudierons comment la folie d'Emma est produite par l'aberration du système colonial dont le pouvoir s'exerce sur des individus qui ne sont pas considérés comme des êtres humains et qui sont contrôlés et assujettis dans le but de les exploiter, système qui, comme le disait Frantz Fanon, était une usine à fabriquer des patients d'hôpitaux psychiatriques.
\end{abstract}

Mots clés: Colonialisme; Amériques; Biopouvoir; Femmes; Folie

Resumo: Este trabalho propõe uma leitura da obra Livre d'Emma, romance de Marie-Célie Agnant, publicado em 2001, que foi objeto de muitos estudos críticos seguindo diversas abordagens, particularmente pós-colonial e feminista. Entretanto, a loucura e a institucionalização de Emma requerem atenção especial. Iremos nos concentrar, portanto, nesses aspectos do romance, situando-o no contexto da reflexão foucaultiana sobre o biopoder e no da loucura no discurso literário. Estudaremos como a loucura de Emma é produzida pelo absurdo do sistema colonial, cujo poder é exercido sobre indivíduos que não são considerados seres humanos e que são controlados e subjugados para serem explorados. Um sistema que, como dizia Frantz Fanon, era uma fábrica de pacientes de hospitais psiquiátricos.

Palavras-chave: Colonialismo; Américas; Biopoder; Mulheres; Loucura

Mais je meurs avant le temps, c'est pour moi grand profit, je le déclare. Quand on vit, comme je le fais, au milieu des maux, comment la mort ne serait-elle pas un avantage?

Antigone (Sophocle: 38$)^{1}$

Le Livre d'Emma raconte l'histoire d'une néomontréalaise d'origine haïtienne, Emma qui, accusée d'avoir tué son bébé, une petite fille, est enfermée dans un hôpital psychiatrique pour être évaluée afin de déterminer si elle est capable de subir un procès judiciaire ou si elle doit être considérée comme non responsable de ses actes en raison de sa santé mentale. La voix narratrice qui structure le roman est celle de Flore, une interprète engagée par le psychiatre d'Emma pour traduire du créole au français les propos de celle-ci, car Emma refuse de parler français ${ }^{2}$.

Au fil du temps, les entretiens entre les deux femmes se font de plus en plus intimes et le psychiatre est laissé de côté. C'est lors de ces tête-à-tête qu'Emma raconte à Flore sa propre histoire et celle de ses ancêtres, des femmes esclaves, leur asservissement, leur dégradation au rang de choses, leurs vies réduites à une existence de bête de somme, considérées par le système juridique comme des biens meubles.

La vie d'Emma est la continuation de celle de ses ancêtres. Elle répète donc leurs mêmes gestes. Ainsi, le refus de parler la langue du colon rappelle celui de son

\footnotetext{
1 La phrase "la folie qui serait venue dans les flancs des bateaux négriers." est une citation du roman de Marie-Célie Agnant Le Livre d'Emma, p. 8.

2 Cet article a été publié originairement dans Récit littéraire, corps biomédical et citoyenneté au Canada - The Literary Narrative, the Biomedical Body and Citizenship in Canada, dirigé par Daniel Laforest et publié dans les Presses universitaires de Limoges (PULIM), 2015.
} 
arrière grande-aïeule, Kilima/Rose, qui pris la décision d'avaler "sa langue et jura de ne plus jamais prononcer un seul mot en présence d'un homme, tant et aussi longtemps qu'elle demeurerait esclave" (AGNANT, p. 134). Le refus d'Emma de parler français et sa détermination à aliéner de plus en plus le psychiatre est un acte d'affirmation de soi et de révolte contre la langue et la culture des colons qui ont infligé tant de supplices aux siens. L'interprète, Flore, passeuse par profession, se rapproche de plus en plus d'Emma en assumant la responsabilité de recevoir et de porter la mémoire des femmes venues d'Afrique dans les Amériques dans les bateaux négriers. L'indicible souffrance de la lignée d'Emma commença sur les côtes africaines lorsque Kilima fut kidnappée par des négriers. Quelques générations plus tard, Emma tuera son bébé fille pour mettre fin à une souffrance qui n'arrête pas de se transmettre de génération en génération, de mère en fille. Comme le souligne Françoise Naudillon, l'infanticide mis en scène par le récit d'Emma appartient à

une tradition qui va de Simone Schwarz-Bart avec Pluie et vent Télumée Miracle (1973) à Tony Morisson avec Tar Baby (1981). La tradition remonte même plus loin dans le temps, car dès le XIXe siècle, des romans mettent en scène des femmes noires, voire des femmes de couleur où l'impossible maternité, l'enfant qui est n'est pas né ou l'enfant mort sont des thèmes sur lesquels s'appuient les schèmes narratifs. (NAUDILLON, p. 77-78)

Emma se suicidera seulement après avoir transmis son savoir à Flore, qui aura la responsabilité de prendre la parole pour raconter une des plus obscures et douloureuses histoires de mémoire de femme: celle de l'esclavage. Comme l'explique Antoinette Marie Sol, le roman "re-présente la blessure de la diaspora africaine (le plus grand déplacement et déracinement humain dans l'histoire du monde) et les atrocités institutionnalisés du système esclavagiste (qui se font sentir ouvertement ou en sourdine dans le racisme contemporain) ainsi que le silence qui entoure les faits de cette histoire" (SOL, p. 969).

Emma avait essayé de rompre ce silence et de rendre publique cette histoire à Bordeaux en présentant une thèse de doctorat dans le monde académique, le filtre socialement reconnu pour déterminer de la validité de ce qui est dit. $\mathrm{Sa}$ recherche se centrait sur le système esclavagiste, la séquestration des hommes et des femmes destinés à l'esclavage et leur transport dans les bateaux négriers. Cependant, comme sa thèse fut refusée, cette histoire ne vit jamais le jour et son livre fut avorté. C'est donc Flore qui devient la garante de la mémoire, le livre d'Emma en quelque sorte, par qui le lecteur prend connaissance d'un passé qui a failli être effacé. Lola (la fille d'Emma) aurait dû porter la mémoire de ses ancêtres. Mais puisqu'Emma avait décidé de finir avec la souffrance de sa lignée, c'est à Flore de prendre le flambeau.

Le filicide commis par Emma est un geste d'une telle violence que, dans le contexte de la culture occidentale moderne, ne peut être expliqué que par la perte de la raison de la mère criminelle. Or, dans la littérature, ce sujet a été traité depuis la Grèce ancienne avec le mythe de Médée qui mit fin à la vie de ses enfants pour leur épargner une vie de souffrances ${ }^{3}$ et pour se venger de son mari, Jason. Au fil du temps, d'autres Médées suivront, presque toutes rongées par une douleur intolérable. L'histoire des femmes esclaves filicides, tel que le cas raconté dans Le livre d'Emma, invite aussi à une lecture politique de ce geste lorsqu'il est contextualisé dans l'histoire du colonialisme et de l'esclavage. Dans le récit de Flore, Emma, en tant que narratrice intradiégétique, prend souvent la parole pour lui transmettre son savoir, hérité de sa grande tante Mattie. Bien qu'Emma parle souvent de son enfance et de ses expériences personnelles, notamment du rejet de sa mère Fifie, elle ne raconte ni ne commente jamais la mort de son enfant. La seule explication que propose le récit d'Emma est la prédiction de type oraculaire annoncée par Mattie:

Comme dans un songe très ancien, tu répéteras les gestes des femmes du clan. Ces gestes qu'elles faisaient pour mettre leurs enfants à l'abri des garrots qui les étouffaient dans les cales des négriers et dans les champs de canne... La dernière goutte de sang du clan de Kilima, déportée vers le Nouveau Monde, s'éteindra avec toi, comme un œil qui ferme. Tout cela n'est pas très clair au fond de moi. Il y a comme un nuage mauve, un gros nuage qui embrouille tout. (AGNANT, p. 136-137)

Ce passage, aux accents réalistes merveilleux, suggère qu'Emma se trouvait dans un état d'aliénation quand elle tua son bébé. Également, dans le présent diégétique, ses gestes et attitudes rappellent le comportement d'une personne traumatisée. De ce point de vue, une des questions soulevées par le roman est : quel est l'environnement, quelles sont les circonstances qui ont provoqué ce traumatisme et occasionné l'état d'aliénation dans lequel Emma a tué son enfant?

D'une part, les études de Michel Foucault portant sur les technologies modernes du biopouvoir procurent le cadre théorique approprié pour tenter certaines explications. D'autre part, les études sur la folie comme topos dans le champ littéraire nous aideront à situer la

\footnotetext{
À ce propos, on peut lire l'excellente étude de Marie Carrière "Trois exils, trois femmes, trois Médées du Québec contemporain" où elle affirme que ces femmes, qui sont victimes d'une "dépression ravageuse" nous obligent "à tenir compte de toute l'humanité de la mère filicide." (CARRIËRE, p. 55)
} 
"folie" d'Emma dans le contexte du discours littéraire et de la société québécoise, ce qui apportera des nuances importantes à notre lecture.

\section{Le corps comme force de travail}

Les systèmes esclavagistes ont employé un éventail de techniques qui leur permettaient l'exploitation des corps bien avant le développement et la mise à point des technologies modernes d'assujettissement des corps, lesquelles, selon Michel Foucault, ont été mises en place et perfectionnées notamment à partir de l'arrivée de la bourgeoisie au pouvoir. Toutefois, malgré le fait que l'esclavage est aussi vieux que le monde et que les études de Foucault se centrent surtout sur la société bourgeoise, celles-ci permettent de comprendre les agencements de ces techniques qui réduisent la vie - humaine - à une simple force de travail.

Foucault explique que le pouvoir disséminé des sociétés contemporaines s'est organisé autour de l'assujettissement des corps. Il appelle cette forme d'exercice du pouvoir "le biopouvoir", lequel pourrait être expliqué comme un ensemble complexe de techniques qui cherchent à maîtriser les corps. Cette maîtrise s'applique tant aux corps physiques des individus, lesquels sont disciplinés et dressés par et pour le travail, qu'à l'humanité qui, en tant qu'espèce, est objet de soins sanitaires. Le souci de l'hygiène, de l'hérédité, de la dégénérescence et, avec lui, le projet eugéniste découlent de cette volonté de contrôle des corps. Foucault explique que les deux aspects visés par les gouvernements pour contrôler les corps sont le sang et la sexualité:

Il est arrivé, dès la seconde moitié du XIXe siècle, que la thématique du sang ait été appelée à vivifier et à soutenir de toute une épaisseur historique le type de pouvoir politique qui s'exerce à travers les dispositifs de sexualité. Le racisme se forme en ce point (le racisme sous sa forme moderne, étatique, biologisante): toute une politique du peuplement, de la famille, du mariage, de l'éducation, de la hiérarchisation sociale, de la propriété, et une longue série d'interventions permanentes au niveau du corps, des conduites, de la santé, de la vie quotidienne ont reçu alors leur couleur et leur justification du souci mythique de protéger la pureté du sang et de faire triompher la race. (FOUCAULT, 1976, p. 197) ${ }^{4}$

Ainsi, le corps assujetti est le lieu de l'exercice d'un pouvoir dont les mécanismes s'adressent non seulement à lui, mais aussi "à la vie, à ce qui la fait proliférer, à ce qui renforce l'espèce, sa capacité de dominer, ou son aptitude à être utilisée" (FOUCAULT, 1976, p. 194). Dans les sociétés contemporaines, c'est la politique du peuplement qui contrôle "ce qui fait proliférer" la vie.
Or, dans le système esclavagiste, même si les corps des femmes étaient exploités pour engendrer de nouveaux esclaves, il n'y avait pas de politiques de famille (bien au contraire) et encore moins une valorisation de la maternité comme nous la concevons de nos jours ou comme elle a été comprise par la société bourgeoise, notamment à partir du XVIIIe siècle. Au contraire, les femmes esclaves étant obligées de se reproduire de manière brutale, la procréation (et non la maternité) leur était imposée. Comme l'explique Emma, leur ventre était nécessaire "pour porter les bras qui servaient à couper cette canne et récolter le coton" (AGNANT, p.25) et leurs corps servaient "à noyer la rage et la violence de toutes les brutes, Nègres et Blancs" (AGNANT, p. 25).

Dans ce contexte, l'infanticide, comme l'explique Antoinette Marie Sol, est un acte de révolte total, au même titre que le suicide, car l'esclave s'appropriait du capital du maître:

La mort de ces enfants innocents est d'autant plus traumatique que délibérée et voulue. L'infanticide dans le contexte historique de l'esclavage est, à l'origine, un acte de générosité et de ravissement, d'euthanasie et de vol. Tuer un enfant destiné à l'esclavage recèle des motivations complexes et contradictoires, comme Toni Morrison l'a si bien montré dans son roman Beloved (1987). Les deux faces de l'infanticide sont de l'amour (épargner un être cher et innocent des horreurs de la servitude) et de la haine (du système esclavagiste, du maître, de soi). (SOL, p. 971)

Faut-il crier au scandale si les femmes esclaves posaient ce geste extrême? Si elles n'étaient que corps et que leurs corps et ceux de leurs enfants n'étaient qu'un meuble, un bien, du capital, comment auraient-elles pu s'aimer et aimer leurs enfants?

\section{Le corps: entre la vie et la vie humaine}

Comme le signale Giorgio Agamben, le biopouvoir, avec ses technologies modernes qui "prennent la vie pour cible" (FOUCAULT, 1976, p.200), s'exerce sur deux aspects de la vie: sur la vie comme zoé ou la vie pure, nue (comme celle des animaux) et sur la bio ou la vie au sein

\footnotetext{
4 Michel Foucault, l'Histoire de la sexualité I, La volonté de savoir, p. 197. Or, une des conséquences de cette évolution du pouvoir a été le nazisme: "Le nazisme a sans doute été la combinaison la plus naïve et la plus rusée - et ceci parce que cela - des fantasmes du sang avec les paroxysmes d'un pouvoir disciplinaire. Une mise en ordre eugénique de la société, avec ce qu'elle pouvait comporter d'extension et d'intensification des micropouvoirs, sous le couvert d'une étatisation illimitée, s'accompagnait de l'exaltation onirique d'un sang supérieur; celle-ci impliquait à la fois le génocide systématique des autres et le risque de s'exposer soi-même à un sacrifice total. Et l'histoire a voulu que la politique hitlérienne du sexe soit restée une pratique dérisoire tandis que le mythe du sang se transformait, lui, dans le plus grand massacre dont les hommes pour l'instant puissent se souvenir", p. 197
} 
de la communauté humaine (AGAMBEN, p.9). Or, le corps existe au sein d'un champ politique dans lequel opèrent des rapports qui l'obligent à tenir certains comportements, dont le travail. L'utilisation économique du corps, employé comme force de production, est alors le résultat d'un investissement politique complexe qui implique sa domination, son dressage. Ainsi, "le corps ne devient force utile que s'il est à la fois corps productif et corps assujetti" (FOUCAULT, 1975, p. 34). Les technologies du biopouvoir qui assurent l'assujettissement sont multiples, diffuses et "rarement formulées" (FOUCAULT, 1975, p. 34), s'adressant à "l'âme" des individus. Car le biopouvoir est un "pouvoir à normes" organisées autour de la loi mais qui la débordent et la dédoublent. Il s'exerce donc doublement par le "dressage disciplinaire des corps-machines et [par la] régulation des flux des corps-espèces" (GARDOU, p. 137).

Il faut donc distinguer entre le simple fait d'être en vie et le fait d'appartenir à la collectivité humaine. $\mathrm{Au}$ cours de l'histoire, la grande majorité des corps assujettis ont été exclus de la vie politique (dans le sens grec de participation à la vie de la polis), c'est-à-dire des cercles dominants. Dans le cas des esclaves, on peut affirmer que même leur appartenance à l'espèce humaine était remise en question ${ }^{5}$. En effet, comme le signale Giorgio Agamben en faisant référence aux détenus des camps de concentrations nazis, la remise en question de la qualité de l'homme remet en question son appartenance à l'espèce humaine (AGAMBEN, p. 18, nous reviendrons sur ce point plus tard). Puisque les esclaves étaient considérés comme de biens meubles (même par la loi, comme dans le Code noir), ils faisaient l'objet d'un commerce qui "rapportait gros", selon les explications d'Emma qui raconte que lors "du temps des négriers et de la canne, les négresses vivantes intéressaient les négociants. On [les] échangeait contre des armes à feu, contre les pierres à fusil, contre les armes blanches, l'alcool, les métaux" (AGNANT, p. 25).

Malgré leur réification et la négation de leur humanité, le travail des esclaves était essentiel au bien-être de ceux qui se considéraient comme les "vrais humains", c'està-dire, essentiel à la prospérité des colons et de leurs empires. Comme l'explique Emma, il fallait "de la bonne sueur de négresse pour féconder la canne, le coton, le tabac" (AGNANT, p. 25). L'aberrante exploitation qui caractérise le système esclavagiste fut possible grâce à la mise en place de mécanismes de pouvoir punitifs très complexes qui allaient de l'enfermement, la surveillance permanente, l'imposition de chaînes, à la torture et à la mutilation des corps. Au moindre signe de révolte ou d'insoumission, l'esclave était brutalement puni. Et si c'était une femme, de surplus elle était violée, même si elle était une enfant. Ce fut le cas de Kilima, l'ancêtre d'Emma qui était convoitée par son propriétaire. Sa protectrice Cécile avait essayé de la défendre pour lui épargner le sort de sa propre fille, Tamu, qui, étant presque une enfant, était décédée suite à un viol. Mais face à la force de ces hommes armés et enragés, les femmes et les esclaves subissaient, impuissants, les tortures les plus cruelles:

Les deux autres se saisirent de Cécile, lui tranchèrent les mains et les pieds. Puis ils s'emparèrent de Kilima et lui coupèrent le bout du nez en lui disant: "Dorénavant, plus personne ne te regardera." Il s'agissait là d'une mutilation qu'on réservait principalement aux femmes. Cette nuit-là sur la plantation, la folie était bien vivante, présente, concrète. (AGNANT, p. 156)

Suite à ce viol, Kilima donnera naissance à une petite fille qu'elle essayera de tuer. Elle sombrera ensuite dans la folie. Ce passage montre d'une part que la cruauté extrême des plantations était en soi une "folie" et que cette même folie/cruauté engendrait plus de blessures, plus de "folie" chez les victimes.

\section{L'âme, prison du corps ${ }^{6}$}

Comme il a été dit plus haut, les technologies de dressage des corps s'adressent à "l'âme" des individus, non à l'âme chrétienne, mais à cette réalité où, selon Foucault, s'articulent les effets du pouvoir et qui est une pièce essentielle pour l'assujettissement du corps. Le dressage du corps, en effet, passe par une intériorisation des normes. En ce sens, Agamben signale que "le processus de subjectivation qui conduit l'individu à s'attacher à sa propre identité et à sa propre conscience, et par conséquent à un pouvoir de contrôle extérieur" (AGAMBEN, p. 13), fait partie des technologies $d u$ soi (subjectives) qui, combinées avec de procédures de totalisation objective, assurent le contrôle du corps. Ce mécanisme d'identification prend place dans ce que Foucault appelle "l'âme", laquelle est produite

à l'intérieur du corps par le fonctionnement d'un pouvoir qui s'exerce sur ceux qu'on punit - d'une façon générale sur ceux qu'on surveille, qu'on dresse et corrige, sur les fous, les enfants, les écoliers, les colonisés, sur ceux qu'on fixe à un appareil de production et qu'on contrôle tout au long de leur existence. (FOUCAULT, 1975, p. 38)

\footnotetext{
Cette question est très complexe, car pour pouvoir fabriquer l'altérité dans le but de se servir de certains individus comme de bêtes de somme en évoquant leur "infériorité" ou pour pouvoir exterminer des catégories entières d'individus, comme les Juifs dans le cas du nazisme, il faut élaborer une hiérarchisation selon laquelle seul le groupe dominant est complètement humain. Cette hiérarchisation détermine l'appartenance et la non-appartenance au groupe. Les femmes par exemple ont été exclues de l'humain non seulement pendant le Moyen Âge (lorsqu'on se demandait si elles avaient ou pas une âme), mais aussi, comme nous le verrons plus loin, à l'époque des Grecs anciens.

6 Michel Foucault, Surveiller et punir. Naissance de la prison, p. 38.
} 
Cette "âme réelle", "née de procédures de punition, de surveillance, de châtiment et de contrainte" (FOUCAULT, 1975, p. 38), est l'instrument du pouvoir politique.

Mais, qu'est-ce qui se passe lorsque l'individu refuse le dressage et ne peut ou ne veut intérioriser les normes? Antigone est un bon exemple de ce refus: elle défie le pouvoir de l'État et se dresse contre l'autorité du tyran allant, comme l'illustre la citation en exergue, jusqu'à préférer la mort pour ne pas vivre "au milieu des maux". Le choix d'Antigone est politique et a un propos: elle sait ce qu'elle fait, pourquoi elle le fait et quelles sont les conséquences de son acte aussi bien pour elle que pour sa parenté. Non seulement elle choisit la désobéissance et la révolte, mais elle s'affirme dans sa condition à la fois humaine (elle parle) et non-humaine. En effet, Antigone n'est pas humaine parce qu'à cause de sa condition de femme les sphères publique et politique - qui font de l'homme un être humain - lui sont interdites.

La situation d'Emma, gardienne de la mémoire des esclaves, est similaire. Effectivement, comme le signale Judith Butler, les esclaves, aussi bien que tant d'autres groupes de populations sans citoyenneté, peuvent être classifiés comme "humains" seulement dans le sens figuré du terme. Bien que leurs vies n'aient pas été détruites par un génocide comme celui perpétré par les Nazis ${ }^{7}$, elles ne sont pas légitimes aux yeux de la communauté, "là où des modèles de reconnaissance permettent la conquête de l'humanité" (BUTLER, p. 90). Cette exclusion renvoie à une mise en question de leur humanité, comme si ces individus ne possédaient pas toutes les qualités nécessaires pour l'être. À Thèbes, Antigone est protagoniste de cette exclusion de l'humain, car dans l'espace commun, là 'où l'humain se constitue à travers les mots et les actes [...], [1]es esclaves, les femmes et les enfants, tous ceux qui n'étaient pas des mâles possédants n'étaient pas autorisés à pénétrer la sphère publique dans laquelle l'humain était constitué par ses actes linguistiques" (BUTLER, p. 91). Antigone, comme Emma et ses ancêtres esclaves,

ne fait pas partie de l'humanité, mais elle parle son langage. Interdite d'agir, elle agit néanmoins, et son acte n'est guère une simple assimilation d'une norme existante. [...] Elle parle dans le cadre d'un langage de pouvoir d'où elle est exclue, s'inscrivant de ce fait dans un langage de revendication avec lequel aucune identification n'est pour finir possible. Si elle est humaine, alors l'humain est entré en métaphore: nous ne connaissons plus son usage propre. Et dans la

\footnotetext{
7 Bien que le projet des Nazis fût l'extermination totale des Juifs (système génocidaire) et que le but de l'esclavage est l'exploitation des corps, il y a certains parallélismes qui peuvent être établis par rapport au racisme et aux expériences de vie à l'intérieur des plantations et des camps de concentration. Aimé Césaire, entre autres, a signalé certaines similitudes dans sa préface à Esclavage et colonisation de Victor Schoelcher.
}

mesure où elle occupe le langage qui ne pourra jamais lui appartenir, elle fonctionne comme un chiasme dans le vocabulaire des normes politiques. (BUTLER, p. 91)

Même si Emma est une femme fortement éduquée, elle est exclue du dialogue social lorsque sa tentative de prise de la parole par le biais de la thèse de doctorat échoue au moment où le jury refuse sa thèse. En ce sens, elle explique à Flore:

C'est avec Mattie que j'ai commencé à comprendre ce qui s'était passé à nord des négriers. [...] Mais après avoir quitté Mattie, ma soif de comprendre est devenue encore plus forte. J'ai fouillé dans les grands livres. J'ai tant cherché, Flore, si tu savais tout ce que j'ai pu lire. Des années entières j'ai cherché, pour découvrir la source de l'horreur de cette haine. (AGNANT, p.117)

Emma, porteuse de la mémoire de ses ancêtres et de l'histoire des femmes esclaves, cherche à valider ses connaissances dans le monde académique par le biais de la recherche, mais en refusant son apport, on lui interdit de participer au dialogue social et l'histoire des siens est bannie de l'Histoire humaine. C'est que, comme l'explique Mattie, "Il y a ceux qui prétendent que nos histoires ne sont que légendes. Ils prétendraient n'importe quoi pour bannir ce temps de leur mémoire" (AGNANT, p. 154). Ce rejet de la mémoire des esclaves vient confirmer leur statut inhumain. Face au refus de sa thèse, elle se sent rejetée à l'extérieur de la frontière de l'humain et avec elle, ses ancêtres le sont aussi aussi, encore une fois. D'autre part, puisque les colons, ou leurs descendants, ne reconnaissent pas leur crime, le dialogue qui aurait ouvert un espace vers la guérison des blessures est interdit. Les victimes de la traite, ou leurs descendants, sont condamnés à revivre la blessure de génération en génération: "Comme quoi ce temps des négriers, ce temps de la canne, jamais ne devait finir" (AGNANT, p. 111).

\section{La folie}

Le fou inspire la peur, le mépris, il est considéré comme capable du pire, mais surtout, il est jugé par la société comme un être "anormal". Or, le concept de folie a évolué au fil du temps, tout comme celui d'anormalité. En réalité, on est fou, normal ou anormal par rapport à quelque chose, dans un contexte déterminé par certains préjugés ou idées acceptées par la majorité. De la même manière, on est déviant par rapport à certaines règles ou habitudes. Par exemple, l'homosexuel est déviant ou anormal pour une société qui considère qu'être hétérosexuel est le comportement approprié afin de fonder des familles, assurer la reproduction, etc., sans remettre en question l'histoire de ce préjugé ou le contexte des 
politiques du sang et de la sexualité qui contrôlent les corps tout en évoquant le bienêtre de l'espèce (Foucault, 1975, 1976). De la même manière, le fou, ayant échoué à sa tentative d'adaptation au fonctionnement de la société, est jugé anormal parce que sa conduite diffère de celle de la majorité des gens qui se pensent "normaux". Il est alors écarté et souvent interné.

C'est en ce sens que l'histoire d'Emma invite à reconsidérer le concept de la folie, car elle remet en question la capacité d'empathie et d'amour des sociétés modernes, leur capacité à construire de nouveaux espaces qui pourraient inclure les blessures du passé ${ }^{8}$. La folie a toujours été présente dans la littérature québécoise. Toutefois, son visage a évolué au fil du temps, au rythme des idéologies ou des préoccupations dominantes dans la société. En ce sens, Robert Viau signale qu'être fou au XIXe siècle était souvent le résultat d'un comportement jugé immoral, c'était donc une forme de punition. À partir de la Révolution Tranquille, les personnages fous, ou les "fous de papier" comme il les appelle, perdent la raison comme conséquence de l'hostilité et de l'injustice du milieu dans lequel ils évoluent.

De ce fait, c'est la société qui est remise en question et considérée comme irraisonnable. Viau explique que "cette folie réactive se rattache aux courants antipsychiatriques. Le fou est en réalité un homme sain dans un monde devenu fou. Il refuse l'indifférence et le conformisme" (VIAU, p. 222) ${ }^{9}$. Ce contexte social brise les esprits sensibles, ceux des visionnaires et des clairvoyants. La rebelle Emma et ses ancêtres, comme tant d'autres héros québécois, ont sombré dans la folie pour les mêmes raisons. Mattie avait averti à Emma du prix à payer pour vivre au milieu des injustices:

On peut emprisonner ton corps, petite, [...] mais promets-moi que ton âme sera toujours libre comme un oiseau. Tu peux la lancer dans les nuages, l'accrocher à la branche la plus haute, sur le pic le plus élevé des montagnes, et l'entendre te parler de toutes parts quand tu es seule. Trop de femmes ne savent pas qu'elles ont une âme. Quand le corps est considéré comme la poussière du chemin sur laquelle on crache, $[\ldots]$ quand tout cela nous arrive, que les yeux ne peuvent retenir les larmes, eh bien, oui, l'âme s'en va, elle s'en va dans les larmes, elle nous quitte comme s'en vont les alluvions. Nous cheminons alors comme des zombies, la folie dans le regard et dans le ventre. (AGNANT, p. 130-131)

Dans le contexte du présent d'Emma (une société moderne et occidentale), une fois que l'esprit est brisé, que l'âme est partie, les révoltés sont déclarés fous par le système médical qui les écarte en les cachant dans des institutions dont les murs garantissent la sécurité des individus normalisés et surtout le bon fonctionnement de la société malsaine. Comme le souligne Viau, ces fous qui diffèrent "de la masse qui suit aveuglément les directives des autorités" sont "des trouble-fête qui, parce qu'ils posent des questions gênantes et des gestes non conformistes, sont séparés de la société et internés" (VIAU, p. 222). Comme Emma, les fous de la deuxième moitié du XXe siècle de la littérature québécoise sont souvent des révoltés, voire des révolutionnaires, des êtres inquiets et inquiétants qui ne peuvent ou ne veulent se conformer aux règles de la société. En conséquence, à partir de la Révolution tranquille "[1]e fou n'est plus un paria, un débauché, mais se transforme en un homme révolté, au sens camusien du terme, conscient de ses responsabilités œuvrant pour son bien-être et celui de ses compatriotes, souvent contre et dans la société" (VIAU, p. 223).

Cette folie spécifique a une longue histoire dans la littérature occidentale. Nous pourrions par exemple citer les cas de Don Quichotte ${ }^{10}$ ou celui de l'Antigone de Sophocle, où la folie varie suivant les points de vue apportés par les différents personnages. De ce fait, un personnage déclaré fou devient le détenteur de la sagesse et de la vérité et vice-versa. Dans la pièce de Sophocle, le premier à parler de folie est Créon qui qualifie Ismène et Antigone d"“insensées": "Ces deux enfants sont insensées: je le dis bien haut! L'une est devenue folle depuis peu; l'autre le fut dès sa naissance" (SOPHOCLE, p. 42). Cependant, après l'intervention du devin Tirésias, qui dénonce la folie de Créon, et des suicides qui se succèdent, il est évident que c'est le comportement aberrant de Créon qui a provoqué le malheur de sa famille et de Thèbes. C'est donc lui le fou. Or, comme le montre la citation en exergue, la souffrance occasionnée par son comportement est telle qu'Antigone préfère mourir, se sentant incapable de vivre "entourée de maux".

La lecture d'Antigone permet de comprendre plusieurs aspects de la folie. D'abord que la folie est relative, car on est déclaré fou par quelqu'un qui a le pouvoir de le

\footnotetext{
Le neuropsychiatre Boris Cyrulnik, qui retrace l'histoire de la psychiatrie et de l'antipsychiatrie, parle des "âmes blessées".

$9 \mathrm{Au}$ moment de la publication du Livre d'Emma, le traitement et la perception des personnes souffrant de maladies mentales avait changé. Nonobstant, dans l'univers du roman, l'hôpital psychiatrique et surtout le psychiatre (qui représente le refus de communiquer avec l'autre) sont présentés dans l'optique de l'antipsychiatrie. À ce sujet, on peut consulter l'entrevue accordée par Marie-Célie Agnant à Florence Ramond Jurney (2005)

10 Don Quichotte est le dernier chevalier aux prises dans une société où il n'y avait plus de place ni pour lui ni pour Dulcinée. Point de géants non plus, rien que le gros bon sens de Sancho. L'Humanisme triomphant aura eu compte du Moyen Âge et de sa pensée magique. Don Quichotte est "fou" parce qu'il ne peut vivre dans un monde de pensée concrète et pratique, mais sa folie sera remise en question selon les lecteurs et selon les époques. Il est important aussi de souligner que cet Humanisme qui a chassé l'imaginaire chevaleresque se nourrissait des anciennes civilisations grecque et romaine et a donné naissance au Siècle des Lumières qui a porté l'ordre bourgeois au pouvoir. À ce sujet, on peut consulter l'article de Fernando de Toro "Don Quijote como "deconstrucción" de modelos narrativos".
} 
faire, ensuite, qu'une personne risque de perdre la raison lorsque la souffrance est insupportable et, finalement, que les injustices sociales et la société consentante sont plus insensées que les personnes étiquetées comme "folles". Dans Le livre d'Emma, Mattie, la voix de la sagesse, avait prévenue Emma des mécanismes de fabrication de la folie destructrice qui guette "les malheureuses":

le monde nous pousse dans la marge jusqu'à nous faire haïr notre propre chair, c'est difficile à comprendre et à accepter, Emma. Il n'est pas étonnant qu'au bout de ce tunnel nous guette la démence, et c'est alors que nous détruisons notre propre chair, parce que nous tremblons pour elle, nous savons ce qui l'attend. (AGNANT, p. 107)

Dans la littérature québécoise, comme le signale Robert Viau, c'est à partir des années 1960 que les fous sont présentés comme des êtres antisociaux, étant ceux qui présentent "un comportement social jugé comme étant perturbateur"11. (VIAU, p. 212) Le livre d'Emma de Marie-Célie Agnant s'inscrit dans cette tradition littéraire. À partir de la deuxième moitié du XXe siècle et plus précisément à partir de la Révolution Tranquille, moment qui coïncide avec l'apparition de l'antipsychiatrie, les fous de la littérature québécoise sont des fous politiques. Dans les récits québécois des années de l'antipsychiatrie, tout comme dans l'Antigone de Sophocle, c'est "l'aliénation collective qui est dénoncée" (VIAU, p. 270). Ismène, sœur d'Antigone, formule cette idée clairement: "Jamais, ô roi, les malheureux ne conservent la raison qu'ils tiennent de la nature: elle succombe sous les épreuves" (SOPHOCLE, p.42). Le propos d'Ismène rendent responsable le tyran (contexte social et politique) d'avoir fait sombrer dans la folie "les malheureux". Au Québec, cette folie prend des formes différentes selon le mouvement idéologique duquel elle prend source ${ }^{12}$.

À partir des années 1980 d'autres voix viendront s'ajouter au dialogue de la société québécoise: celles des écrivains venus d'ailleurs dont la production littéraire est désignée par la critique comme "littérature migrante". Certains romans publiés par ces écrivains mettent en scène des personnages migrants et leurs expériences en tant que nouveaux arrivants. La littérature québécoise s'est enrichie d'histoires autres qui présentent des personnages dont les bagages charrient de souvenirs d'autres cieux. Le livre d'Emma s'inscrit dans ce contexte. L'histoire d'Emma et celle de ses ancêtres est une histoire haïtienne; ses aïeules, considérées comme du "bois d'ébène", descendirent des bateaux négriers et les récits qui ont marqué son enfance et sa jeunesse sont peuplés de corps exploités et mutilés par les colons, des horreurs des plantations, des trahisons et d'abandons.
L'histoire d'Emma nous invite ainsi à réfléchir sur l'importance de guérir les blessures du passé, sans quoi, nous serons condamnés à le revivre à l'infini. En effet, comme le disait Frantz Fanon, il est nécessaire d' "inventer" une nouvelle humanité13, guérir des blessures laissées par l'exploitation, la misère, la corruption, la guerre. Il faut oser "inventer", découvrir un nouveau jour, il faut créer des espaces autres, chercher un dialogue dans un espace qui soit un cheminement. Dans le cas du Québec et du Canada, les exemples d'écriture migrante qui illustrent l'insertion des "temps-autres" dans le dialogue culturel sont nombreux. Les voix des victimes qui ont échappé à la terreur des dictatures latino-américaines et à d'autres horreurs sont intégrées au dialogue social et grâce à leurs histoires, elles transforment les cultures.

Mais ce moment de transition n'est pas facile à vivre, car l'écrivain venu d'ailleurs vit dans un état d'incertitude, dans un sentiment d'exclusion et d'exil. Homi Bhabha nomme cet état un être unhomely, condition paradigmatique et postcoloniale exposée dans les fictions qui négocient le pouvoir des différences culturelles. Bhabha signale que la condition de unhomely est traumatisante ${ }^{14}$. C'est une sorte de maison incomplète des origines raciales et culturelles qui établit un lien avec le «entre» diasporique et produit des symboles pour construire une "vraie maison" de sorte que, dans l'avenir, les familles fragmentées qui habitent la diaspora aient des attachements. Dans ce processus se développe une "intimité interstitielle" où, à leur tour, les divisions binaires des sphères opposées dans l'espace social se rejoignent. Ces sphères de la vie sont reliées entre elles par une temporalité intermédiaire qui produit une image autre du monde et de l'histoire. L'intimité, ce moment interstitiel, se traduit esthétiquement par des récits qui représentent l'hybridité, la différence à l'intérieur d'une société, d'un groupe, d'une nation. Ce sont des récits qui mettent en scène un sujet qui habite la fissure de l' "entre" de la réalité. L'inscription de cette existence limitrophe crée des images qui jettent un pont entre le chez soi et le monde (BHABHA, 1994).

\footnotetext{
${ }^{11}$ Viau explique que le rôle du psychiatre est de "défendre les gens normaux, ou plutôt normatifs, au détriment des anormaux, des gêneurs, qui deviennent rapidement des "fous" dès qu'ils sont classifiés comme tels." (VIAU, p. 212)

${ }^{12}$ Robert Viau explique que les trois mouvements où elle s'enracine sont les "mouvement de libération des peuples colonisés, mouvement antinucléaire et mouvement de libération des femmes. "(VIAU, p. 221)

${ }^{13}$ Il affirme en effet: "Si nous voulons que l'humanité avance d'un cran, si nous voulons la porter à un niveau différent de celui où l'Europe l'a manifestée, alors, il faut inventer, il faut découvrir. [...] Pour l'Europe, pour nous-mêmes et pour l'humanité, camarades, il faut faire peau neuve, développer une pensée neuve, tenter de mettre sur pied un homme neuf. "Frantz Fanon, Les damnés de la terre, p. 376.

${ }^{14}$ Dans l'entrevue accordée par Marie-Célie Agnant à Florence Ramond Jurney, on peut constater le sentiment de non-appartenance de l'auteure, qui affirme ne pas avoir "d'appartenance territoriale" (Ramond Jurney, p. 390).
} 
Ce pont est certainement jeté entre l'auteure, MarieCélie Agnant, et la société québécoise. Nonobstant, au niveau du récit, l'histoire Emma met en relief justement l'impossibilité de bâtir des ponts quand la parole est niée. C'est le psychiatre qui doit traiter Emma -et qui ne comprend pas son histoire- le personnage qui illustre le mieux cette impossibilité. Comme l'explique Lesley Curtis, le respect de l'opacité de l'autre (ici celle d'Emma) aurait débouché dans une Relation dans le sens glissantien du terme:

L'existence du passé dans le présent fait que ceux qui ne reconnaissent pas ses effets continus trouvent le passé qui hante Emma trop difficile à comprendre, trop opaque. Dans le roman, le désir de comprendre, de réduire, de même nier l'opacité de l'histoire (qui fait aussi partie de l'opacité d'Emma) est lié à la Transparence, la réification et donc la déshumanisation des milliers de victimes de la traite. Cette déshumanisation existe toujours et continue à créer et à soutenir une hiérarchie des cultures. Comme l'opacité empêche la déshumanisation, elle devient un outil au présent. Si l'on accepte l'opacité de l'Autre, si l'on admet son existence sans juger, sans essayer de comprendre et donc de déstructurer sa différence, on arrive à la coexistence glissantienne des opacités. Cette Relation basée sur l'opacité permet deux choses: l'existence des humanités en empêchant une hiérarchisation des cultures différentes et une acceptation de la présence continue du passé. (CURTIS, p. 68)

Dans le roman la Relation glissantienne est impossible parce que le docteur MacLeod est un mur humain qui provoque l'incommunication. Il n'est pas seulement la figure de l'autorité et du pouvoir à l'intérieur des murs de l'hôpital psychiatrique, mais son comportement rappelle celui des membres du jury qui refusèrent la thèse (le droit à la parole) d'Emma. Dans le présent du récit, le docteur MacLeod rétablit la hiérarchie dominateur-dominé. Emma refuse de lui parler, car elle refuse le statut de subalterne que le médecin lui attribue. Comme d'autres personnages de la littérature québécoise, elle préfère le monologue, l'incohérence, voire passer comme folle pour ne pas plier à son jeu. C'est que, comme l'explique Robert Viau, "[1]'incohérence du fou et du terroriste brise la cohérence de la domination et établit de nouveaux rapports, inquiétants, à base d'hésitations, de monologues incompréhensibles" (VIAU, p. 233). Il ajoute que plus le fou est incompréhensible et incohérent, plus il est dangereux et marque la distance avec la raison dominante qu'il remet en question.

Emma, haïtienne d'origine, échouée dans un hôpital psychiatrique, remet en question le désintérêt et l'indifférence des sociétés occidentales modernes vis-à- vis la tragédie de l'esclavage. Mais malgré son histoire autre au sein de sa société d'accueil, dans le contexte de la littérature québécoise, Emma appartient à la race de Menaud, le héros du roman de Félix-Antoine Savard Menaud, maître-draveur (1937), premier personnage de la littérature québécoise à devenir fou de douleur à cause des injustices et des abus des colonisateurs. Comme le signale Viau, "Menaud, vieux et brisé, est le reflet d'un Québec vendu et trahi" (VIAU, p. 224). Emma aussi devient folle sous le poids de l'héritage du colonialisme qui a détruit les cultures, les familles et l'esprit des hommes et des femmes victimes de ce système. En ce sens, Frantz Fanon observait:

La colonisation, dans son essence, se présentait déjà comme une grande pourvoyeuse des hôpitaux psychiatriques. [...] Parce qu'il est une négation systématisée de l'autre, une décision forcenée de refuser à l'autre tout attribut d'humanité, le colonialisme accule le peuple dominé à se poser constamment la question: "Qui suis-je en réalité"? (FANON, p. 189)

Les séquelles de ce système perdurent jusqu'à nos jours ${ }^{15}$. On consigne, entre autres, des problèmes économiques, la honte de sa race, le ressentiment, l'anxiété collective, la difficulté à former des familles parce que les hommes "disparaissent" et que la figure du père est typiquement absente. En ce sens, Emma décrit les descendants mâles de la traite comme des "fantômes de malheur qui hantent notre île, se promènent de maison en maison pour déposer leurs œufs dans les entrailles des négresses et se sauver, le pantalon en bataille" (AGNANT, p. 110).

La tragédie d'Emma est de devoir porter l'histoire occultée de ses ancêtres esclaves sans réussir à la révéler ni à faire reconnaître l'injustice. Elle explique en effet que l'origine de sa folie se trouve dans le manque de reconnaissance, car lorsqu'elle a essayé de révéler l'histoire sans y avoir réussi, elle a "flanché", étant donné que "la route a été trop longue, $[. .$.$] la course trop$ éperdue" (AGNANT, p. 110). L'expérience d'Emma est similaire à celle des autres "fous de papier" québécois. En ce sens, Robert Viau signale que dans le contexte de la société "impassible et indifférente" dans laquelle évoluent ces personnages, l'individu révolté "s'épuise en paroles et en actes. Après une courte période d'extase, il n'a plus la force de riposter au sphinx social qui le réduit au silence" (VIAU, p. 225).

Emma, qui vit obsédée par un passé duquel elle n'a pas pu se débarrasser, tuera sa fille pour lui épargner le sort de devenir une négresse, un objet, dont la vie ne

\footnotetext{
15 En ce sens, Frantz Fanon affirme: "Et nous aurons à panser des années encore les plaies multiples et quelques fois indélébiles faites à nos peuples par le déferlement colonialiste." (FANON, p. 189)
} 
sera que larmes et souffrances. Comme l'affirme Marie Carrière, Emma "porte une blessure incommensurable, héritée de Fifie et de ses grands-mères, ce qui la rend incapable de vivre [...]" (CARRIÈRE, p. 55). Emma est à la fois "folle et trop lucide" (AGNANT, p.33), comme Antigone, comme Menaud, comme tant d'autres fous de la littérature dont la folie/lucidité renvoie à la révolte, au refus de vivre dans un entourage qui nie leur humanité. Emma lutte pour ses droits, pour la reconnaissance de son humanité et celle des siens. Elle se révolte contre les injustices du présent et du passé des sociétés racistes et oppressives qui repoussent vers l'extérieur des marges de l'humain de millions d'individus. Comme le souligne Robert Viau, "[1]'oppression nomme et crée la folie qui, par ce fait même, s'exaspère et s'irrite contre elle. La folie naît d'un double phénomène de rejet ayant des causes extrinsèques et intrinsèques. Elle résulte en monologue, en silence armé de part et d'autre" (VIAU, p. 246).

Subséquemment, à l'instar de la réflexion de Robert Viau sur les fous des romans québécois, nous pouvons affirmer qu'Emma, comme Menaud et les autres, "sont des générateurs de conscience" et que "[m]algré leur échec personnel, ils suscitent des épigones qui compléteront la tâche entreprise. Le dernier épisode est loin d'être terminé" (VIAU, p.231). Dans le roman de Marie-Célie Agnant, Flore sera la passeuse de la mémoire, la responsable de transmettre l'histoire d'Emma et de ses ancêtres qui sont dorénavant les siennes, car elle porte leur mémoire.

$$
* * *
$$

Lorsqu'Emma répète le geste de tant d'esclaves la précédant, qui ont tué leurs enfants pour leur épargner une vie de tourments et qu'elle se suicide, elle cherche à mettre fin à sa souffrance ancestrale et en même temps elle se dérobe à une société qui l'a aliénée. Aliénation qui fut déclenchée par une vie fragilisée par la douleur transmise de génération en génération et brisée par la rigidité de la société moderne qui refuse de l'écouter et de l'intégrer à son dialogue. Pour exister en tant qu'humain, il faut que la vie soit politique en ce sens qu'elle doit être action racontée aux autres. Car, comme le signalait Hannah Arendt, le récit, en tant qu'action partagée, est la première dimension dans laquelle l'individu vit sa vie humaine (politique). Dans le cadre de son internement, le rejet de son médecin réaffirme le rejet d'Emma à l'extérieur de l'humain, ce qui scelle son statut de paria, d'exclue, de fantôme. Emma finira sa vie noyée, littéralement, sous le poids des larmes. Contrairement au Sisyphe camusien, Emma aura lâché son rocher, car pour elle, lorsque la vie humaine n'est pas possible, la vie tout court ne l'est pas non plus.
Or, Le livre d'Emma a été publié dans une culture qui accueille des histoires autres en les intégrant au dialogue social. En ce sens, Marie-Célie Agnant, comme tant d'autres écrivains dits migrants, invite à réfléchir sur le passé, ses blessures et l'importance de les traiter. Car même si l'histoire a eu lieu ailleurs, la société d'accueil ouvre l'espace nécessaire au dialogue. Cette réflexion sur les blessures du colonialisme découvre aussi l'inquiétude du présent et du futur de la vie humaine au sein des sociétés capitalistes qui utilisent les corps comme des outils de production. Cette inquiétude se présente face à l'aliénation occasionnée par le biopouvoir et face aux aberrantes conséquences du contrôle du sang et de la sexualité.

Cette histoire autre de la littérature québécoise qui raconte l'histoire d'"une folie qui serait venue dans les bateaux négriers", fait partie d'une esthétique qui incorpore les voix des écrivains qui produisent un discours bio-fictif en recréant leur traversée, ce qui met en évidence la complexité de la nouvelle société: hétérogène, multiple, en mouvance, en perpétuelle rénovation, une société où les relations s'établissent de manière rhizomatique, en négociant, en créant une culture qui est devenir, un procès jamais achevée.

Ainsi, l'histoire d'Emma avec son lourd bagage colonial invite à une relecture du propre passé colonial, à revisiter le douloureux héritage que raconte l'histoire de Menaud. D'ailleurs, au Canada, le peuple québécois n'est pas le seul à avoir souffert des atrocités du système colonialiste. L'histoire d'Emma, sa folie, sa solitude, son enfermement, sa révolte, sa marginalité, nous invitent à réfléchir aussi sur les conséquences des Écoles résidentielles (ou Pensionnats indiens), sur les vies brisées des individus qui, même des générations plus tard, souffrent encore les effets des atrocités commises au nom du progrès et de la civilisation.

\section{Références}

AGAMBEN, Giorgio. Homo sacer I. Le pouvoir souverain et la vie nue. Paris: Seuil, 1997.

AGNANT, Marie-Célie. Le Livre d'Emma. Montréal: Les éditions du remue-ménage, 2002.

ARENDT, Hannah. La condition de l'homme moderne. Paris: Calmant-Lévi, 1961.

BHABHA, Homi. The Location of Culture. London and New York: Routledge, 1994.

BUTLER, Judith. Antigone: la parenté entre vie et mort. Paris: EPEL, 2003.

CARRIÈRE, Marie. Trois exils, trois femmes, trois Médées du Québec contemporain, dans Atlantis, v. 35, n. 1, p. 47-58, 2010.

CÉSAIRE, Aimé. Introduction, dans Victor Schoelcher, Esclavage et colonisation. Paris: PUF, 1948. (Collection de textes choisis). 
CURTIS, Lesley. Vite elle se referme: L'opacité, dans Le Livre d'Emma de Marie-Célie Agnant, dans Women in French Studies, volume 21, 2013. p. 68-78.

CYRULNIK, Boris. Les âmes blessées. Paris: Odile Jabob, 2014.

DE TORO, Fernando. Don Quijote como "deconstrucción" de modelos narrativos, dans Cervantes, su obra y su mundo: Actas del I Congreso Internacional sobre Cervantes (Historia de la literatura española desde sus fuentes). Madrid: Edi, 1981. p. 635-655.

FANON, Frantz. Les damnés de la terre. Paris: François Maspero, 1961.

FOUCAULT, Michel. Surveiller et punir. Naissance de la prison. Paris: Gallimard, 1975.

FOUCAULT, Michel. Histoire de la sexualité I, La volonté de savoir. Paris: Gallimard, 1976.

GARDOU, Charles. Fragments sur le handicap et la vulnérabilité. Paris: Érès, 2005.

GLISSANT, Édouard. Poétique de la relation. Poétique III. Paris: Gallimard, 1990.
NAUDILLON, Françoise. Le continent noir des corps: représentation du corps féminin chez Marie-Célie Agnant et Gisèle Pineau, dans Études françaises, v. 41, n. 2, p. 73-85, 2005 , $<$ http://id.erudit.org/iderudit/011379ar>.

RAMOND JURNEY, Florence. Entretien avec Marie-Célie Agnant, The French Review, v. 79, n. 2, p. 384-394, déc. 2005 SALA-MOLINS, Louis. Le code noir. Paris: PUF, 1997.

SAVARD, Félix-Antoine. Menaud, maître-draveur. 1937.

SOL, Antoinette Marie. Histoire(s) et traumatisme(s): l'infanticide dans le roman féminin antillais, dans The French Review, v. 81, n. 5, p. 967-984, Apr. 2008. <http://www.jstor. org/stable/25481325>.

SOPHOCLE. Antigone. Paris: Éditions Larousse, 2012.

VIAU, Robert. Les fous de papier. Montréal: Éditions du Méridien, 1989.

Recebido: 25 de abril de 2015

Aprovado: 25 de junho de 2015

Contato: marentsen@ustboniface.mb.ca 